\title{
意大利小麦真菌传棒状病毒与欧洲小麦花叶 病毒和土传黑麦花叶病毒属同一种病毒"
}

\section{杨建平 ${ }^{(12)}$ 陈剑平 ${ }^{(1 * *}$ 陈 畑 ${ }^{(1)}$ 程 华 ${ }^{(1)}$ M. J. Adams ${ }^{(4)}$}

(1)浙江省农业科学院病毒室, 杭州 310021; (2)浙江大学农业与生物技术学院, 杭州 310029; (3)浙江大学生命科学学院, 杭州 310027; (4)Plant Pathology Department, IACR-Rothamsted, Harpenden, Herts AL5 2JQ, UK)

\begin{abstract}
摘要 测定了从意大利硬红小麦上分离的一个真菌传棒状病毒分离物两个 RNA 的 核苷酸全序列, 并与美国的土传小麦花叶病毒(SBWMV)、最近发表的从法国小麦上分 离的欧洲小麦花叶病毒(EWMV)和从德国黑麦和小麦上分离的 3 个土传黑麦花叶病毒 (SBRMV-C,-G, -O)进行了序列比较和系统树分析. 结果表明, 意大利分离物的 RNA1 和 RNA2 分别由 7025 和 3688 个碱基组成, 与 EWMV 的同源性分别为 $97.5 \%$ 和 $98.6 \%$, 与 SBRMV-G 为 $95.5 \%$ 和 $85.8 \%$, 而与 SBWMV 仅为 $70.6 \%$ 和 $64.5 \%$. 因此, 意大利 分离物与 SBWMV 不同, 但与 EWMV 和 SBRMV 同属一种病毒, 建议将这些欧洲病 毒命名为土传禾谷类花叶病毒(SBCMV), 以避免与以前的名字混淆.
\end{abstract}

\section{关键词真菌传棒状病毒属 核苷酸序列 分类学}

真菌传棒状病毒属成员为正链单链 RNA 病毒, 其两个 RNA 分别被包裹在长度为 300 和 $150 \mathrm{~nm}$ 的棒状粒子中, 并由土壤中根肿菌纲的禾谷多黏菌(Polymyxa graminis)传播. 该属的代 表种是土传小麦花叶病毒(SBWMV). SBWMV 最初在美国报道 ${ }^{[1]}$, 目前在美国绝大多数冬小 麦区、法国、意大利、日本、中国、埃及和巴西均有发生 ${ }^{[2]}$. Shirako 等人 ${ }^{[3]}$ 测定了 SBWMV 内布拉斯加州分离物的两个 RNA 全序列. 真菌传棒状病毒属包括燕麦金色条纹病毒 $(\mathrm{OGSV})^{[4]}$ 和高粱褪绿斑点病毒 $(\mathrm{SCSV})^{[5]}$.

在中国和法国小麦上发生的真菌传棒状病毒一直被认为是 SBWMV. 最近的序列分析表 明它们与 SBWMV 截然不同, 从而建议更名为中国小麦花叶病毒 $(\mathrm{CWMV})^{[6]}$ 和欧洲小麦花叶 病毒 $(\mathrm{EWMV})^{[7]}$. Koenig 等人 ${ }^{[8]}$ 最近测定了从德国南部分离的 3 个真菌传棒状病毒分离物的序 列, 其中分离物 $\mathrm{G}$ 和 $\mathrm{O}$ 来自黑麦, $\mathrm{C}$ 来自小麦. 这些分离物起先也被认为是 SBWMV 的一个 株系 ${ }^{[9,10]}$. 基于它们与北美 SBWMV 序列上的差异, 并且可以侵染黑麦, 被命名为土传黑麦花 叶病毒(SBRMV) ${ }^{[8]}$.

在意大利, 硬红小麦上发生的一种真菌传棒状病毒基于其生物学和血清学特性也被描述 为 $\mathrm{SBWMV}{ }^{[11]}$. 为了澄清意大利分离物的分类地位, 我们测定了该病毒基因组全序列, 并与同

2000-04-24 收稿, 2000-08-29 收修改稿

* 国家自然科学基金(批准号: 39970482)、浙江省人才基金(批准号: RC9604)、浙江省自然科学基金(批准号: 399421)、

欧共体(批准号: INCO CI18 CT96-0049)资助项目

*** 联系人(E-mail: jpchen@public1.hz.zj.cn)

1) 本文报道的核苷酸序列已登录于 DDBJ/GenBank/EMBL 数据库, 登录号为 AJ252151(RNA1)和 AJ252152(RNA2) 
属其他成员的分子关系进行比较.

\section{1 材料与方法}

\section{1 病毒分离物}

具有典型症状的感病硬红小麦(品种, Creso)叶片于 1998 和 1999 年 3 月采自意大利北部 Ozzano 的小麦田中, 经中国国家动植物检疫局批准引进(编号为: 9930040), 在浙江省农业科 学院 $-80^{\circ} \mathrm{C}$ 保存备用. 根据 Shirako 等人 ${ }^{[12]}$ 描述的方法从病叶中提取病毒. 病小麦叶总 RNA 和 病毒 RNA 用 QIAGEN RNeasy Plant Minikit 按产品手册中的流程分别从病叶和病毒提纯制剂 中提取.

\section{2 第 1 链 CDNA 合成和 PCR 扩增}

按 EWMV RNA1 和 RNA2 的 $3^{\prime}$ 端序列 ${ }^{[7]}$ 设计的引物 ISB1-7R(表 1), 能同时与 RNA1 和 RNA2 配对, 以病毒 RNA 为模板进行逆转录合成第 1 链 $\mathrm{cDNA}$, 保存于 $-20^{\circ} \mathrm{C}$ 备用. 逆转录反 应体系为 $25 \mu \mathrm{L} .10 \mu \mathrm{L}$ 病小麦叶总 $\mathrm{RNA}$ 与 $1 \mu \mathrm{L}$ 引物 $\mathrm{ISB} 1-7 \mathrm{R}(800 \mathrm{mg} / \mathrm{mL})$ 混合, $70^{\circ} \mathrm{C}, 10 \mathrm{~min}$, 冰浴. 加入 $4 \mu \mathrm{L} 5 \times$ 逆转录酶缓冲液(Promega), $2 \mu \mathrm{L} 0.1 \mathrm{~mol} / \mathrm{L}$ DTT, $1 \mu \mathrm{L} 10 \mathrm{mmol} / \mathrm{L} \mathrm{dNTPs}$ 和 $1 \mu \mathrm{L} \mathrm{H} \mathrm{H}_{2} \mathrm{O}$ 混匀, 置于 $37^{\circ} \mathrm{C}, 2 \mathrm{~min}$. 然后加入 $1 \mu \mathrm{L}(20 \mathrm{U}) \mathrm{M}-\mathrm{MLV}$ 逆转录酶, 置于 $37^{\circ} \mathrm{C}, 1 \mathrm{~h}$, 最 后加入 $80 \mu \mathrm{L} \mathrm{H} \mathrm{H}_{2} \mathrm{O},-20^{\circ} \mathrm{C}$ 保存备用. 按发表的 EWMV 序列 ${ }^{[7]}$ 设计引物(表 1), 用于意大利分离 物 cDNA1 和 cDNA2(RNA1: 74 7 023 nt, RNA2: 8 3 $686 \mathrm{nt}$ )不同区域 PCR 扩增. PCR 反应体 系为 $50 \mu \mathrm{L}$, 含有 1 或 $2 \mu \mathrm{L}$ 模板 $\mathrm{cDNA}, 20 \mathrm{pmol}$ 配对引物, $200 \mathrm{mmol} / \mathrm{L} \mathrm{dNTP}, 1.5 \mathrm{mmol} / \mathrm{L}$ $\mathrm{MgCl}_{2}, 50 \mathrm{mmol} / \mathrm{L} \mathrm{KCl}, 10 \mathrm{mmol} / \mathrm{L}$ Tris- $\mathrm{HCl}, \mathrm{pH} 8.3$ 和 1 或 $2.5 \mathrm{U}$ Taq DNA 聚合酶 $(\mathrm{BM}$ 或 GIBCO). DNA 模板 $94{ }^{\circ} \mathrm{C}$ 变性 $3 \mathrm{~min}$, 扩增 30 个循环, 每个循环 $94^{\circ} \mathrm{C}$ 变性 $1 \mathrm{~min}$, 在退火温度 $\left(T_{\mathrm{m}}\right.$, 见表 1)下退火 $1 \mathrm{~min}, 72^{\circ} \mathrm{C}$ 延伸 $0.5 \sim 2 \mathrm{~min}$ (按 $1 \mathrm{~kb}$ 需 $1 \mathrm{~min}$ 的合成时间计算). PCR 产物在 $1 \%$ 或 $1.5 \%(w / v)$ 的琼脂糖中电泳.

\section{3 病毒 RNA 末端序列的测定}

用 RACE 的方法扩增病毒 RNA 末端序列 ${ }^{[6,7]}$. 为了得到病毒 RNA1 和 RNA2 的 $3^{\prime}$ 端核 苷酸序列, 在病毒 RNA1 和 RNA2 的 $3^{\prime}$ 端连接上 DNA 引物 ZHM1(5' -CTC TTC CCC TCC CTC CTC-3'，为了提高连接效率, $5^{\prime}$ 端第 1 个 $\mathrm{C}$ 磷酸化). 连接反应的体系为 $25 \mu \mathrm{L}$, 大约 $1.2 \mu \mathrm{g}$ 病毒 RNA, $4 \mu \mathrm{mol} / \mathrm{L}$ ZHM1, $50 \mathrm{mmol} / \mathrm{L}$ Tris-HCl, $\mathrm{pH}$ 7.8, $10 \mathrm{mmol} / \mathrm{L} \mathrm{MgCl}_{2}, 10 \mathrm{mmol} / \mathrm{L}$ DTT, 1 mmol/L ATP, $6 \mu \mathrm{g} / \mathrm{mL}$ BSA, 25\% PEG8000 和 22.5 U RNA 酶抑制剂(Life Technologies Ltd.)和 20 U T4 RNA 连接酶(New England Biolabs), $37^{\circ} \mathrm{C}$ 温浴 30 min. RNA-ZHM1 连接产物用 QIAGEN RNeasy Plant Minikit 按产品手册中的流程纯化. 引物 ZHM2 (5'-GAG GAG GGA GGG GAA GAG-3'，与 ZHM1 互补)分别与 ISB1-7F(RNA1) 和 ISB2-3F (RNA2)配对来扩增病毒 RNA1 和 RNA2 的 $3^{\prime}$ 端序列.

为了得到病毒 RNA1 和 RNA2 的 $5^{\prime}$ 端核苷酸序列, 用纯化的病毒 RNA 作为模板, 用混 合引物 ISB 1-1R(RNA1)和 FCPr(RNA2)来合成第 1 链 cDNA1 和 cDNA2. 杂交分子用 QIAquick Gel Extraction Kit(QIAGEN)纯化, 然后 $100^{\circ} \mathrm{C}$ 变性 $2 \mathrm{~min}$, 迅速置于冰上冷却. 用上述方法在 该单链 $\mathrm{cDNA} 1$ 和 $\mathrm{CDNA} 2$ 连接引物 ZHM1, 然后以此作为模板, 用引物 ZHM2 分别与引物 RFSB1-19(RNA1)和 RFSB2-5(RNA2)配对来扩增 RNA1 和 RNA2 的 5' 序列. 
表 1 用于基因片段 RT-PCR 扩增的引物

\begin{tabular}{|c|c|c|c|}
\hline 引物 & 位置 a) & 序列 & $T_{\mathrm{m}} \mathrm{b}^{2}$ \\
\hline \multicolumn{4}{|c|}{ RNA1 正向(配对)引物: } \\
\hline ISB1-1AF & $74 \sim 89$ & 5' -GCT TCG GCG ATA AAT C-3' & 43 \\
\hline FFSB $1-6^{c)}$ & $831 \sim 850$ & $5^{\prime}$-CGT GTA AGG ATA ATG AGT GG-3' & 53 \\
\hline FFSB $1-14^{c}$ & $1568 \sim 1585$ & 5' -TCA AAA GGC GTT GAG GAG-3' & 49 \\
\hline ISB 1-A4F & $2449 \sim 2464$ & 5' -GTA GCT CTT CTA ACT C-3' & 41 \\
\hline $\mathrm{FS}^{(\mathrm{c})}$ & $3334 \sim 3355$ & 5' -ATG ATA AAT TCA GGG CAG CGA C-3' & 59 \\
\hline ISB $1-5 \mathrm{~F}$ & $3907 \sim 3923$ & 5' -CGA GGC ATA GAC GTT CG-3' & 49 \\
\hline FFSB $1-15^{\mathrm{c}}$ & $4964 \sim 4981$ & $5^{\prime}$-CAG AAC AAA CGG CAT TAC-3' & 47 \\
\hline ISB 1-7F & $5868 \sim 5883$ & $5^{\prime}$-CTG CGT ACT GCT GAA C-3' & 45 \\
\hline ISB $1-3 \mathrm{tF}$ & $6495 \sim 6510$ & $5^{\prime}$-GGC GAA TGT GCA AAA G-3' & 43 \\
\hline \multicolumn{4}{|c|}{ RNA1 反向(互补)引物: } \\
\hline RFSB $1-19^{c)}$ & $466 \sim 449$ & 5' -CCA CCA ATA TCG TAA ACC-3' & 47 \\
\hline ISB 1-1R & $1077 \sim 1062$ & 5' -CTC GCA ACT CTG TCA G-3' & 45 \\
\hline ISB1-2R & $1940 \sim 1925$ & $5^{\prime}$-CCA AGA GTC AAT GAA G-3' & 41 \\
\hline RFSB $1-10^{c}$ & $2609 \sim 2591$ & 5' -AGA CGA ATC GCT CTC TTC C-3' & 53 \\
\hline ISB1-4R & $3435 \sim 3420$ & 5' -GCA CTC GTT TTG CAC C-3' & 45 \\
\hline $\mathrm{RS}^{\mathrm{c})}$ & $4051 \sim 4029$ & $5^{\prime}$-TAC TTC GAC CCA AAC CGT CAT TT-3' & 61 \\
\hline ISB1-5R & $5036 \sim 5021$ & 5' -GTT AGA ACC ATA AGT G-3' & 39 \\
\hline RFSB $1-13^{c}$ & $6158 \sim 6140$ & $5^{\prime}$-TGT CCT TGT TTT TGC GTC C-3' & 51 \\
\hline RFSB $1-12^{\mathrm{c})}$ & $6502 \sim 6481$ & 5' -CAT TCG CCC TAA TCA АAT CAT C-3' & 57 \\
\hline ISB1-7R & $7023 \sim 7007$ & $5^{\prime}$-GGC CGG ATT ACC CTC CG-3' & 53 \\
\hline \multicolumn{4}{|c|}{ RNA2 正向 (配对)引物: } \\
\hline ISB2-1F & $8 \sim 27$ & 5' -CAT TAT CAC GTT ACG TTG AG-3' & 51 \\
\hline FCPf & $312 \sim 326$ & 5' -GTG GTT GTG CAG TTA-3' & 39 \\
\hline FFSB $2-6^{c}$ & $661 \sim 682$ & $5^{\prime}$-GAA ATG CTA TGA TAG GGA CGA C-3' & 59 \\
\hline FFSB2-12 & $2254 \sim 2274$ & $5^{\prime}$-ACA TGC AGG CTC AGA TTA GAC-3' & 57 \\
\hline ISB2-3F & $3164 \sim 3179$ & 5' -GTT CGC CAC GGA AGT G-3' & 47 \\
\hline \multicolumn{4}{|c|}{ RNA2 反向 (互补)引物: } \\
\hline RFSB2-5 $5^{c}$ & $549 \sim 526$ & 5' -TGT GTG TCC CAA ATC TTT TCT GTC-3' & 63 \\
\hline $\mathrm{FCPr}$ & $908 \sim 893$ & $5^{\prime}$-ATC TTC GAC AAT GCG T-3' & 41 \\
\hline RFSB2-3 $3^{c}$ & $1506 \sim 1483$ & 5' -GAT CTG CGA CGA TTA GAG CTT CAC-3' & 67 \\
\hline ISB2-2R & $2474 \sim 2459$ & 5' -CGT GTT GCT AGC AAC G-3' & 45 \\
\hline RFSB2-10 & $3283 \sim 3264$ & $5^{\prime}$-GGT AGG ACA TTT TCG CTA GG-3' & 55 \\
\hline RFSB2-9c) & $3615 \sim 3595$ & 5' -ACC AGT CGC TCC TCA TCA TC-3' & 57 \\
\hline ISB1-7R & $3686 \sim 3670$ & 5' -GGC CGG ATT ACC CTC CG-3' & 53 \\
\hline
\end{tabular}

a) EWMV RNA1(AJ132576)和 RNA2(AJ132577) 序列[7]中的位置; b) $\left.T_{\mathrm{m}}=4(\mathrm{C}+\mathrm{G})+2(\mathrm{~A}+\mathrm{T})-5 ; \mathrm{c}\right)$ 根据文献[7]设计

\subsection{PCR 片段克隆和序列分析}

PCR 产物经纯化后克隆到 pGEM-T(Promega)质粒中, 然后采用 ABI PRISMTM 377 DNA 自动测序仪进行序列测定. 所有 cDNA 均进行正反双向测序, 序列结果用 GCG 等软件进行分 析. 


\section{2 结果}

意大利小麦真菌传棒状病毒的 RNA1 含有 7025 个核苷酸, RNA2 含有 3688 个核苷酸. RNA1 和 RNA2 同真菌传棒状病毒属其他成员具有一样的基因组结构.

RNA1 具有 3 个可读框(ORF). ORF1 编码一个具有甲基转移酶和解旋酶基序，并以 UGA 终止, 该终止子可能被通读而产生一个合并了 ORF2 较大的产物. ORF2 编码的是依赖 RNA 的 RNA 聚合酶( RdRp). ORF3 编码运动蛋白.

RNA2 具有 3 个 ORF. ORF1 编码 $19 \mathrm{ku}$ 的外壳蛋白, UGA 终止子可能被通读而产生一个 合并了 ORF2 较大的产物. 与真菌传棒状病毒属的其他成员一样, 在第 1 个 AUG 上游位于 213 215 位置上有一个符合读框的 CUG 密码子, 被认为能形成一较大的外壳蛋白 (约 $24 \mathrm{ku})^{[13]}$. ORF3 编码一个小的富含半胱氨酸的蛋白质(约 $19 \mathrm{ku}$ ), 其功能不详.

序列分析表明, 意大利分离物、法国 EWMV 和德国 SBRMV 分离物之间除了一些主要 位于末端非编码区有小的差异外，各基因大小彼此几乎完全相同(表 2). 从整个基因组来看, 意大利分离物的 RNA1 和 RNA2 的核苷酸同源性与 EWMV 分别为 $97.5 \%$ 和 $98.6 \%$, 与 SBRMV-G 分别为 $95.5 \%$ 和 $85.8 \%$ ，与美国的 SBWMV 分别为 $70.6 \%$ 和 $64.5 \%$ ，表明意大利病 毒与 EWMV 和 SBRMV 为同一种病毒, 而与 SBWMV 则不同. RNA1(表 3)和 RNA2(表 4)不 同基因区域的核苷酸和氨基酸序列的比较表明，除了 $5^{\prime}$ 端非编码区外，意大利分离物所有基 因区域通常比德国的 SBRMV 更接近 EWMV. 这种相似性在外壳蛋白通读区尤其明显, 而这 个区域一般是基因组中变异最大的部分 ${ }^{[7]}$. 外壳蛋白和 RdRp 氨基酸序列的系统树分析也进一 步证实意大利分离物, EWMV 和 SBRMV 与 SBWMV, OGSV 和 CWMV 有显著的差异(图 1). 与 其他被描述的真菌传棒状病毒一样, 意大利分离物 RNA1 和 RNA2 的 $3^{\prime}$ 端非编码区的核苷 酸序列同源性非常高(91.3\%).

表 2 序列已测定的真菌传棒状病毒属各成员不同基因大小

\begin{tabular}{|c|c|c|c|c|c|c|c|c|}
\hline & 意大利分离物 & EWMV & SBRMV-G & SBRMV-C & SBRMV-O & CWMV & OGSV & SBWMV \\
\hline RNA1 & AJ252 151 & AJ132 576 & AF146 278 & AF146 279 & AF146 280 & AJ012 005 & AJ132 578 & L07 937 \\
\hline $5^{\prime}$ 非编码区 & 107 & 107 & 108 & $84^{*}$ & $84 *$ & 101 & 116 & 101 \\
\hline 复制酶 & 3927 & 3927 & 3927 & 3927 & 3927 & 4053 & 4038 & 3963 \\
\hline 复制酶通读区 & +1524 & +1524 & +1524 & +1524 & +1524 & +1524 & +1524 & +1524 \\
\hline 非编码区 & 61 & 61 & 61 & 61 & 61 & 61 & 51 & 64 \\
\hline 运动蛋白 & 981 & 981 & 981 & 981 & 981 & 990 & 975 & 984 \\
\hline $3^{\prime}$ 非编码区 & 425 & 425 & 425 & $408^{*}$ & $408^{*}$ & 418 & 407 & 463 \\
\hline 全长 & 7025 & 7025 & 7026 & * & * & 7147 & 7111 & 7099 \\
\hline RNA2 & AJ252 152 & AJ132 577 & AF146 281 & AF146 282 & AF146 283 & AJ012 006 & AJ132 579 & L07 938 \\
\hline $5^{\prime}$ 非编码区 & 332 & 327 & 329 & $304^{*}$ & $308 *$ & 323 & 320 & 333 \\
\hline 外壳蛋白 & 531 & 531 & 531 & 531 & 531 & 531 & 531 & 531 \\
\hline 外壳蛋白通读区 & +1734 & +1734 & +1725 & +1734 & +1734 & +1749 & +1521 & +1734 \\
\hline 非编码区 & 69 & 69 & 69 & 69 & 69 & 48 & 48 & 66 \\
\hline 末半胱氨酸蛋白 & 498 & 498 & 498 & 498 & 498 & 522 & 522 & 525 \\
\hline 3' 非编码区 & 524 & 524 & 521 & $503 *$ & $503 *$ & 396 & 290 & 404 \\
\hline 全长 & 3688 & 3683 & 3673 & * & * & 3569 & 3232 & 3593 \\
\hline
\end{tabular}

* 近全长序列 
表 3 欧洲小麦真菌传棒状病毒和土传小麦花叶病毒 RNA1 不同基因之间氨基酸 (右上, 斜体)和核苷酸(左下)同源性 ${ }^{\mathrm{a}}$

\begin{tabular}{|c|c|c|c|c|c|c|}
\hline & 意大利分离物 & EWMV & SBRMV-G & SBRMV-C & SBRMV-O & SBWMV \\
\hline \multicolumn{7}{|l|}{$5^{\prime}$ 非编码区 } \\
\hline EWMV & 94.4 & $*$ & & & & \\
\hline SBRMV-G & 96.2 & 97.2 & $*$ & & & \\
\hline SBRMV-C & 97.2 & 96.3 & 98.1 & $*$ & & \\
\hline SBRMV-O & 97.2 & 96.3 & 98.1 & 100.0 & $*$ & \\
\hline SBWMV & 74.3 & 69.3 & 75.2 & 80.2 & 80.2 & $*$ \\
\hline \multicolumn{7}{|l|}{ 复制酶 } \\
\hline 意大利分离物 & $*$ & 97.8 & 95.8 & 95.1 & 95.3 & 75.6 \\
\hline EWMV & 97.1 & $*$ & 96.0 & 95.5 & 95.7 & 75.5 \\
\hline SBRMV-G & 95.2 & 95.1 & $*$ & 95.6 & 95.8 & 75.4 \\
\hline SBRMV-C & 94.5 & 94.3 & 95.1 & $*$ & 98.9 & 75.5 \\
\hline SBRMV-O & 94.7 & 94.6 & 95.4 & 99.0 & $*$ & 76.4 \\
\hline SBWMV & 69.9 & 69.7 & 69.6 & 69.6 & 69.6 & $*$ \\
\hline \multicolumn{7}{|l|}{ 复制酶通读区 } \\
\hline 意大利分离物 & $*$ & 99.0 & 98.2 & 97.4 & 97.2 & 82.9 \\
\hline EWMV & 98.4 & $*$ & 97.6 & 96.9 & 96.7 & 82.3 \\
\hline SBRMV-G & 96.2 & 96.2 & $*$ & 98.6 & 98.4 & 83.7 \\
\hline SBRMV-C & 96.3 & 96.1 & 97.0 & $*$ & 99.0 & 84.1 \\
\hline SBRMV-O & 96.0 & 95.9 & 96.6 & 99.2 & $*$ & 83.5 \\
\hline SBWMV & 74.9 & 74.5 & 74.8 & 75.1 & 75.1 & $*$ \\
\hline \multicolumn{7}{|l|}{ 运动蛋白 } \\
\hline 意大利分离物 & $*$ & 98.2 & 95.7 & 95.7 & 95.4 & 70.6 \\
\hline EWMV & 97.7 & $*$ & 96.0 & 96.0 & 95.1 & 70.2 \\
\hline SBRMV-G & 95.1 & 95.8 & $*$ & 96.3 & 94.8 & 70.2 \\
\hline SBRMV-C & 95.2 & 96.1 & 95.8 & $*$ & 97.9 & 70.2 \\
\hline SBRMV-O & 94.8 & 95.5 & 95.0 & 99.0 & $*$ & 69.3 \\
\hline SBWMV & 67.4 & 67.3 & 68.1 & 67.6 & 66.9 & $*$ \\
\hline \multicolumn{7}{|l|}{ 3' 非编码区 } \\
\hline EWMV & 98.6 & $*$ & & & & \\
\hline SBRMV-G & 97.2 & 97.2 & $*$ & & & \\
\hline SBRMV-C & 97.9 & 97.4 & 97.2 & $*$ & & \\
\hline SBRMV-O & 98.0 & 97.5 & 97.3 & 99.8 & $*$ & \\
\hline SBWMV & 72.4 & 72.9 & 73.6 & 69.7 & 68.9 & $*$ \\
\hline
\end{tabular}

a) EWMV, SBRMV-G, -C, -O 和 SBWMV 的序列分别引自文献 $[3,7,8]$ 
表 4 欧洲小麦真菌传棒状病毒和土传小麦花叶病毒 RNA2 不同基因之间氨基酸 (右上, 斜体)和核苷酸(左下)同源性 ${ }^{\mathrm{a}}$

\begin{tabular}{|c|c|c|c|c|c|c|}
\hline & 意大利分离物 & EWMV & SBRMV-G & SBRMV-C & SBRMV-O & SBWMV \\
\hline \multicolumn{7}{|l|}{ 5' 非编码区 } \\
\hline EWMV & 96.3 & $*$ & & & & \\
\hline SBRMV-G & 91.5 & 91.7 & $*$ & & & \\
\hline SBRMV-C & 95.1 & 95.1 & 91.8 & $*$ & & \\
\hline SBRMV-O & 97.6 & 96.3 & 93.9 & 95.4 & $*$ & \\
\hline SBWMV & 58.5 & 60.6 & 59.6 & 63.6 & 62.2 & $*$ \\
\hline \multicolumn{7}{|l|}{ 外壳蛋白 } \\
\hline 意大利分离物 & $*$ & 97.7 & 96.6 & 98.3 & 98.9 & 80.2 \\
\hline EWMV & 97.7 & $*$ & 95.5 & 97.2 & 97.7 & 79.0 \\
\hline SBRMV-G & 89.6 & 89.1 & $*$ & 98.3 & 96.6 & 81.4 \\
\hline SBRMV-C & 96.6 & 95.1 & 90.4 & $*$ & 98.3 & 81.9 \\
\hline SBRMV-O & 98.1 & 96.2 & 89.5 & 95.9 & $*$ & 80.2 \\
\hline SBWMV & 74.0 & 72.9 & 74.8 & 75.5 & 73.3 & $*$ \\
\hline \multicolumn{7}{|l|}{ 外壳蛋白通读区 } \\
\hline 意大利分离物 & $*$ & 99.1 & 77.9 & 95.2 & 97.8 & 60.4 \\
\hline EWMV & 99.3 & $*$ & 77.5 & 94.6 & 97.2 & 59.9 \\
\hline SBRMV-G & 77.4 & 77.7 & $*$ & 78.2 & 77.9 & 63.5 \\
\hline SBRMV-C & 94.6 & 94.5 & 77.4 & $*$ & 95.3 & 59.2 \\
\hline SBRMV-O & 96.9 & 96.9 & 77.5 & 93.8 & $*$ & 60.1 \\
\hline SBWMV & 63.4 & 63.1 & 63.9 & 62.8 & 63.1 & $*$ \\
\hline \multicolumn{7}{|c|}{ 富含半胱氨酸蛋白 } \\
\hline 意大利分离物 & $*$ & 99.4 & 98.8 & 98.8 & 99.4 & 63.3 \\
\hline EWMV & 99.0 & $*$ & 98.2 & 98.2 & 98.8 & 63.0 \\
\hline SBRMV-G & 96.4 & 96.6 & $*$ & 98.8 & 99.4 & 63.9 \\
\hline SBRMV-C & 96.4 & 96.6 & 98.0 & $*$ & 99.4 & 63.9 \\
\hline SBRMV-O & 98.2 & 98.4 & 96.6 & 96.2 & $*$ & 63.9 \\
\hline SBWMV & 70.7 & 70.3 & 70.7 & 69.9 & 70.3 & $*$ \\
\hline \multicolumn{7}{|l|}{ 3' 非编码区 } \\
\hline EWMV & 98.1 & $*$ & & & & \\
\hline SBRMV-G & 94.8 & 95.2 & $*$ & & & \\
\hline SBRMV-C & 94.6 & 95.4 & 98.8 & $*$ & & \\
\hline SBRMV-O & 97.8 & 98.4 & 95.4 & 95.0 & $*$ & \\
\hline SBWMV & 55.8 & 55.5 & 60.1 & 58.5 & 63.0 & $*$ \\
\hline
\end{tabular}

a) EWMV, SBRMV-G, -C, -O 和 SBWMV 的序列分别引自文献 [3, 7, 8] 
$\operatorname{RdRp}$

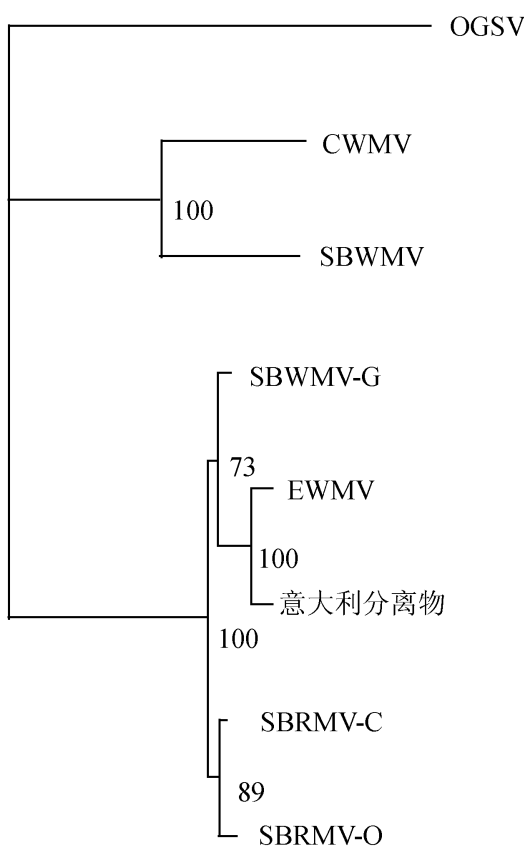

外壳蛋白

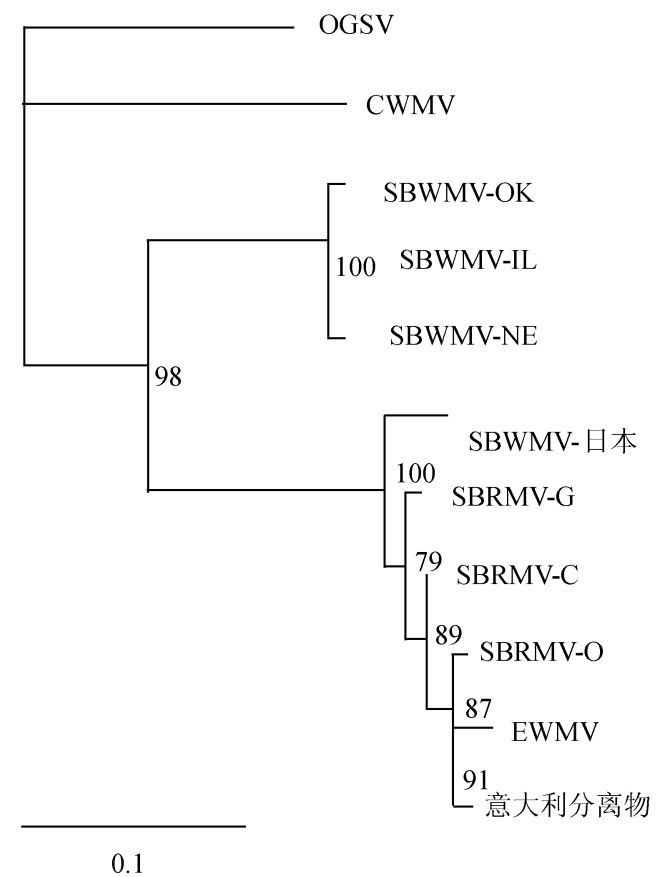

0.1

图 1 意大利小麦真菌传棒状病毒与其他真菌传棒状病毒 RNA 依赖的 RNA 聚合酶(RdRp) 和外壳蛋白氨基酸序列的系统树(树根为 OGSV) CWMV, EWMV, OGSV, SBWMV 和 SBRMV 的序列分别引自文献[3, 6, 7, 8]

\section{3 讨论}

由上述结果可见，意大利小麦上分离的真菌传棒状病毒、法国小麦上的 $\mathrm{EWMV}^{[7]}$ 和德国 黑麦和小麦上的 $\mathrm{SBRMV}^{[8]}$ 的核苷酸序列非常相似. 这些分离物应属同一种病毒, 而与 SBWMV, OGSV 和 CWMV 有明显的区别. 日本 SBWMV 外壳蛋白基因与欧洲分离物具有一 定的同源性 ${ }^{[13]}$, 但有关日本 SBWMV 的分类尚需要进一步序列数据才能确定. 尽管意大利分 离物、法国 EWMV 和德国的 SBRMV-C 分离物均来自小麦, 而 SBRMV-G 和 SBRMV-O 来 自黑麦, 但分析表明它们不存在与寄主有关的分子差异, 且显然是同一种病毒. 在与 $R$. Koenig 博士探讨后, 我们认为对这些病毒分离物应该另起一个名字, 它既不暗示特殊的地理 区域(欧洲), 又不专指较小的寄主范围(黑麦和小麦). 土传禾谷类花叶病毒(soil-borne cereal mosaic virus, SBCMV)满足这些要求.

致谢 感谢意大利 Bologna 大学 C. Rubies-Antonell 博士提供意大利病毒分离物; 本实验 室つ爱坡和张恒木提供部分引物; 德国联邦农林科学院 R. Koenig 博士与作者一起讨论病毒 命名. 


\section{参考文献}

1 Brakke M K. Soil-borne wheat mosaic virus. Association of Applied Biologists Descriptions of Plant Viruses, 1976, 77: 4

2 Brakke M, Langenberg W G. Experiences with soil-borne wheat mosaic virus in North America and elsewhere. In: Cooper J I, Asher M J C, eds. Developments in Applied Biology II. Viruses with Fungus Vectors. Warwick: Association of Applied Biologists, 1988. 183 202

3 Shirako Y, Wilson T M A. Complete nucleotide sequence and organization of the bipartite genome of soil-borne wheat mosaic virus. Virology, 1993, 195: 16 32

4 Plumb R T, Catherall P L, Chamberlain J A, et al. A new virus of oats in England and Wales. Ann Phytopathol, 1977, 9: $365 \sim 370$

5 Kendall T L, Langenberg W G, Lommel S G. Molecular characterization of sorghum chlorotic spot virus, a proposed furovirus. J Gen Virol, 1988, 69: 2 335 2 345

6 Diao A, Chen J, Ye R, et al. Complete sequence and genome properties of Chinese wheat mosaic virus, a new furovirus from China. J Gen Virol, 1999, 80: 1 141 1 145

7 Diao A, Chen J, Gitton F, et al. Sequences of European wheat mosaic virus and oat golden stripe virus and genome analysis of the genus Furovirus. Virology, 1999, 261: 331 339

8 Koenig R, Pleij C W A, Huth W. Molecular characterization of a new furovirus mainly infecting rye. Arch Virol, 1999, 144: $2125 \sim 2140$

9 Huth W, Lesemann D E. Wheat soil-borne mosaic virus isolated from rye in Germany. In: Koenig R, ed. Proceedings of the First Symposium of the International Working Group on Plant Viruses with Fungal Vectors (Shriftenreihe der Deutschen Phytomedizinischen Gesellschaft 1). Stuttgart: Ulmer, 1990. 139 141

10 Huth W, Lesemann D E. Fungus-transmitted soil-borne virues on rye in Germany. Z Pfanzenkr Pflanzenschutz, 1996, 103: $120 \sim 124$

11 Rubies-Autonell, Vallega C V. Observations on a mixed soil-borne wheat mosaic virus and wheat spindle streak mosaic virus infection in durum wheat (Triticum durum Desf.). J Phytopathol, 1987, 119: 111 121

12 Shirako Y, Brakke M K. Two purified RNA's of soil-borne wheat mosaic virus are needed for infection. J Gen Virol, 1984, 65: $119 \sim 127$

13 Shirako Y. Non-AUG translation initiation in a plant RNA virus; a forty-amino-acid extension is added to the N terminus of the soil-borne wheat mosaic virus capsid protein. J Virol, 1998, 72: $1677 \sim 1682$ 